\title{
CASE OF HYPERACTIVE CHILD
}

\author{
Harparam Singh Ghuman ${ }^{1}$
}

1Medical Officer, Johal Hospital, Ramamandi, Jalandhar.

\section{ABSTRACT}

\section{BACKGROUND}

Attention Deficit Hyperactivity Disorder (ADHD) is a mental disorder of the neurodevelopment type. ${ }^{1}$ It is characterised by problems paying attention, excessive activity or difficulty controlling behaviour which is not appropriate for person's age. These symptoms begin by age six to twelve, are present for more than six months and cause problems in at least two settings (such as school, home or recreational activities). ${ }^{2}$

\section{KEYWORDS}

ADHD, Differential Diagnosis, Hyperactive, Hyperkinetic.

HOW TO CITE THIS ARTICLE: Ghuman HS. Case of hyperactive child. J. Evolution Med. Dent. Sci. 2017;6(22):1823-1825, DOI: 10.14260/Jemds/2017/401

\section{BACKGROUND}

Inattention, hyperactivity (restlessness in adults), disruptive behaviour and impulsivity are common in ADHD. Academic difficulties are frequent, as are problems with relationships. ${ }^{3}$ The symptoms can be difficult to define, as it is hard to draw a line at where normal levels of inattention, hyperactivity and impulsivity end and significant levels requiring interventions begin. In children, problems paying attention may result in poor school performance. Although it causes impairment, particularly in modern society, many children with ADHD have a good attention span for tasks they find interesting. ${ }^{4}$ Child can be easily distracted forget things, and frequently switch from one activity to another. They have difficulty maintaining focus on one task, become bored with a task after only a few minutes unless doing something enjoyable. They seem to not be listening when spoken to. They sometimes dash around, touching or playing with anything and everything in sight. They can be very impatient and often interrupt conversations or others' activities. ${ }^{5}$

\section{CASE REPORT}

A 9-year-old boy presented to the Paediatric Outpatient Department with the complaint of headache for about 2 years. He was referred to rule out any pathology to Neurology Department for related causes of headache. A detailed history was obtained, which reveals the chronic headache was bitemporal, dull aching, intermittent in nature, not associated with nausea or vomiting. He was suffering from loss of sleep at night, just sleeping for $4-6$ hrs. in night with daytime restlessness and irritability. He has trouble completing or turning in homework assignments, often losing things (e.g. pencils, toys, assignments) needed to complete tasks or activities. He loses temper easily, mood fluctuations and abnormal body movements.

Financial or Other, Competing Interest: None.

Submission 28-01-2017, Peer Review 02-03-2017,

Acceptance 09-03-2017, Published 16-03-2017.

Corresponding Author:

Dr. Harparam Singh Ghuman,

Medical Officer,

Johal Multi-speciality Hospital,

Jalandhar.

E-mail: baljitbajwa7@gmail.com

DOI: $10.14260 /$ jemds $/ 2017 / 401$

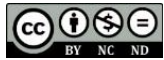

He was born by normal vaginal delivery with spontaneous cry and breathing. There was a mild delay in attaining mental and motor milestones. At 5 years of age, he was diagnosed of having ADHD and treated for 8 months following which he had a good improvement in academics and behaviour. At 7 years of age, he had fractured ankle joint for which fixation was done and pop cast was given. At 8 years of age he suffered from pus discharge from left ear, which leads to his habit of pricking ear with pencil. Subsequently, treatment for the same was done. Audiological evaluation reveals bilateral mild sensory neural hearing loss with $95 \%$ speech discrimination score. A multiprofessional evaluation obtained the final diagnosis as ADHD/bilateral mild sensory neural hearing loss/oppositional defiant disorder/bipolar mood disorder/learning disorder (Especially in mathematics)/overflow motor movements/joint laxity. A multi-professional approach is adopted in its integrative management with satisfactory improvement.

\section{DISCUSSION}

A child who is inattentive, hyperactive with poor academic performance is difficult to manage both at school and at home. With increasing awareness of parents and teachers, the referral rate of such children to a healthcare professional is increasing in the current era. Chances of underdiagnosing and undertreating children with attention deficit is possible due to many overlapping and coexisting features of ADHD with childhood hearing loss, auditory processing disorder, learning disorder, autism, personality disorder, generalised anxiety disorder, bipolar mood disorder, obsessive compulsive disorder, oppositional defiant disorder, conduct disorder, psychotic disorder and low IQ. ${ }^{6}$ A multi-professional team approach is therefore necessary for complete evaluation and comprehensive management.

ADHD is a neurodevelopmental psychiatric disorder manifesting with significant inattention, hyperactivity and impulsive behaviour which is inappropriate to the corresponding age persisting for at least 6 months as per DSMIV-TR criteria (Diagnostic and Statistical Manual of Mental Disorders). It has a prevalence rate of $5-10 \%$ in $2-17$ years of age group children and boys are more commonly affected than girls. Undiagnosed childhood hearing loss can also present with hyperactivity, impulsivity, inattention due to difficulty in hearing instructions or understanding what is expected from the child.7 
Hearing impairment may itself lead to delayed language acquisition, which in turn leads to poor academic performances. Children with mild Sensory Neural Hearing Loss (SNHL) were reported to have significant dysfunction on aspects like social behaviour, communication, academic performance and self-esteem. ${ }^{8}$ Children with acquired sensory neural hearing loss appears to be at increased risk for attention problems. The coexistence of hearing loss with ADHD makes it still more difficult for accurate timely diagnosis and management.

Central Auditory Processing Disorder (CAPD) is characterised by difficulty in processing sounds in the brain without any hearing loss. The overlapping features of ADHD and CAPD are inattention, unfocussed nature, easy distractibility, poor academic skills, unable to follow instructions and perform tasks. ${ }^{9}$ A child with CAPD has difficulty in concentrating in noisy environment; sound discrimination problems; poor auditory, memory and language processing problems. ${ }^{10}$ The coexistence of CAPD with ADHD severely compromises the child's ability to listen, remember and perform tasks. Specific Learning Disorder (LD) is diagnosed when a child's achievement on individually administered standardised testing in reading, writing or mathematics is substantially below that expected for age, schooling and level of intelligence (DSM-IV-TR). LD was reported in $70 \%$ of the children with ADHD. Children with LD and ADHD seems to have more severe attention problems than either of the two presenting alone. ${ }^{11}$

Studies have reported that $50 \%$ of Autism Spectrum Disorders (ASD) population meets the diagnostic criteria for ADHD. ASD coexisting with ADHD leads to significant deficits in social behaviour, adaptive functioning and executive control.12 A variety of psychiatric disorders are reported to be comorbid with ADHD, which includes Oppositional defiant disorder, Conduct disorder, Bipolar mood disorder, Anxiety disorder and Depression. Transient or persistent sleep disorders are also common in ADHD. ADHD with comorbid tic disorders show disruptive behaviour and high functional impairment. ${ }^{13}$ Motor overflow movements are reported in some children with ADHD due to white matter abnormalities in motor and premotor circuits, which are important for motor response inhibition. Higher frequency of epileptiform discharges in EEG is reported in children with over-activity, behavioural problems and SNHL. ${ }^{14-15}$ The prevalence of joint laxity is found to be higher in children with ADHD, gives a new basis for further studies. ${ }^{16}$

Such a complex array of comorbid conditions associated with ADHD can be explained by genetic, environmental, structural and functional changes in the nervous system in causing ADHD.

Genetic alterations related to dopaminergic, noradrenergic and serotonergic neurotransmission systems were reported to have a role in causing ADHD. 17 Chromosomal rearrangements are found to have a significant role in the pathogenesis of syringomyelia and its association with ADHD, developmental delay, cognitive impairment, ASD and seizure disorders were reported. A significant decrease in the volume of white matter and brain structures with focal cortical thinning in prefrontal cortex and altered functional connectivity is seen in ADHD patients. ${ }^{18}$ Maternal illness, very low birth weight, preterm birth, intrapartum complications and neonatal illness lead to a wide spectrum of neurodevelopmental disorders like cerebral palsy, mental retardation, ADHD, learning disorder, language disorder, hearing and visual impairment. ${ }^{19}$ Adult ADHD persists in 10\% - $60 \%$ of children diagnosed with childhood ADHD. A higher risk of degenerative neural disorders, in particular dementia with Lewy bodies is noted to be present in patients with proceeding adult ADHD.20 Such a wide spectrum of neurodevelopmental, psychiatric and other comorbid disorders presenting in attention deficit children requires a multi-professional approach in timely accurate diagnosis and comprehensive management. 21 This includes-

1. Complete history and physical examination.

2. Behavioural assessment test at school and home (Conners' Rating Scales Revised CRS-R, Vanderbilt ADHD Diagnostic Rating Scales).

3. Audiological evaluation (subjective and objective tests) Pure tone audiogram, speech audiometry, impedance audiogram, otoacoustic emissions, brainstem evoked response audiometry as required. Children above 6 years must undergo testing for sound discrimination, auditory memory and language processing to rule out CAPD by determining if the child's ability to listen decreases if the background noise is slowly increased and to the instructions spoken at faster speeds.

4. Language development assessment.

5. Assessment for learning disabilities, personality disorders and psychiatric disorders.

6. Cognitive assessment-IQ testing.

7. Neurological assessment, EEG, brain imaging.

8. Vision testing.

9. Polysomnography for sleep disorders if required.

The role of a developmental paediatrician in identifying children with behavioural abnormalities and motivating the parents on subjecting the children to a multi-evaluation process is vital. Pharmacotherapy is still a main treatment modality for ADHD which has no role in CAPD, SNHL and language disorders. The beneficial effects of stimulants like methylphenidate, dextroamphetamine and pemoline on children with ADHD are proven in reducing the symptoms and improving the academic performance. ${ }^{22}$ The role of an ophthalmologist- to rule out vision impairment; otologist and audiologist- to rule out hearing impairment, CAPD and adequate amplifications with hearing aids if needed; speech language pathologist for language disorders is obvious. The role of a psychiatrist in the assessment of attention deficit children includes assessment for behavioural disorders with behaviour rating scale test, learning disorders, psychiatric disorders, sleep disorders, personality assessment, IQ testing, providing psychoeducation to parents and children, behavioural therapy, cognitive behavioural therapy and pharmacotherapy when needed for psychiatric disorders. ${ }^{23}$ Coexisting seizure disorder, tic disorders and other neurological problems requires a neurologist's intervention in assessment and management.

The role of teachers at school in arranging assistive listening devices and acoustic environmental modifications is necessary to create an optimal listening environment, which is beneficial to children with ADHD and hearing loss. A quiet classroom with FM systems attached reduces the amount of competing background noise, which helps to concentrate and understand speech. Teaching skills with breaking large multistep process to smaller multiple steps, creating a checklist of things to be done using mnemonics to learn steps 
and sequences, incidental learning with stories and skits, using computer programmes and smart boards will be much beneficial.

Parents at home play a key role in seeking prompt medical advice, cooperating for multidisciplinary evaluation, dietary support with sugar restricted, additive-preservative free food rich in fibre, omega-3 fatty acids, iron, folate and zinc which enhances the effectiveness of stimulant therapy in ADHD. Finally, special care and motivation from the parents will help these children to lead a better life in all aspects.

\section{CONCLUSION}

ADHD is diagnosed by an assessment of a person's childhood behavioural and mental development including ruling out the effects of drugs, medications and other medical or psychiatric problems as explanations for the symptoms. It often takes into account feedback from parents and teachers with most diagnoses begun after a teacher raises concerns with such a comprehensive management with a multi-professional approach. Attention deficit children can be successful in school and lead productive lives.

\section{REFERENCES}

[1] Caroline S. Encyclopedia of cross-cultural school psychology. Springer Science \& Business Media 2010:p 133.

[2] Walitza S, Drechsler R, Ball J. The school child with ADHD. Ther Umsch 2012;69(8):467-73.

[3] Willcutt EG. The prevalence of DSM-IV attentiondeficit/hyperactivity disorder: a meta-analytic review. Neurotherapeutics 2012;9(3):490-9.

[4] Weinberg WA, Emslie GJ. Attention deficit hyperactivity disorder: the differential diagnosis. J Child Neurol 1991;6(Suppl):S23-36.

[5] Scahill L, Schwab-Stone M. Epidemiology of ADHD in school-age children. Child Adolesc Psychiatr Clin N Am 2000;9(3):541-55.

[6] Bess FH, Dodd-Murphy J, Parker RA. Children with minimal sensorineural hearing loss: prevalence, educational performance, and functional status. Ear Hear 1998;19(5):339-54.

[7] Kelly DP, Kelly BJ, Jones ML, et al. Attention deficits in children and adolescents with hearing loss. A survey. Am J Dis Child 1993;147(7):737-41.

[8] Kelly D, Forney J, Parker-Fisher S, et al. The challenge of attention deficit disorder in children who are deaf or hard of hearing. Am Ann Deaf 1993;138(4):343-8.

[9] Chermak GD, Somers EK, Seikel JA. Behavioral signs of central auditory processing disorder and attention deficit hyperactivity disorder. J Am Acad Audiol 1998;9(1):78-84.

[10] Moore DR. The diagnosis and management of auditory processing disorder. Lan Speech Hear Serv Sch 2011;42(3):303-8.
[11] Riccio CA, Hynd GW, Cohen MJ, et al. Comorbidity of central auditory processing disorder and attentiondeficit hyperactivity disorder. J Am Acad Child Adolesc Psychiatry 1994;33(6):849-57.

[12] Mayes SD, Calhoun SL, Crowell EW. Learning disabilities and ADHD: overlapping spectrum disorders. J Learn Disabil 2000;33(5):417-24.

[13] Murray MJ. Attention-deficit/hyperactivity disorder in the context of autism spectrum disorders. Curr Psychiatry Rep 2010;12(5):382-8.

[14] Pliszka SR. Comorbidity of attentiondeficit/hyperactivity disorder with psychiatric disorder: an overview. J Clin Psychiatry 1998;59(7):508.

[15] Lycett K, Mensah FK, Hiscock H, et al. A prospective study of sleep problems in children with ADHD. Sleep Med 2014;15(11):1354-61.

[16] Sukhodolsky DG, Scahill L, Zhang H, et al. Disruptive behavior in children with tourette's syndrome: association with ADHD comorbidity, tic severity, and functional impairment. J Am acad Child Adolesc Psychiatry 2003;42(1):98-105.

[17] D’Agati E, Casarelli L, Pitzianti MB, et al. Overflow movements and white matter abnormalities in ADHD. Prog Neuropsychopharmacol Biol Psychiatry 2010;34(3):441-5.

[18] Lance EI, Shapiro BK. Confounding diagnosis in the neurodevelopmental disabilities population: a child with hearing loss, absence epilepsy and attentiondeficit hyperactivity disorder (ADHD). J Child Neurol 2013;28(5):645-7.

[19] Amirsalari S, Radfar S, Ajallouyean M, et al. Prevalence of epileptiform discharges in children with sensorineural hearing loss and behavioral problems compared to their normal hearing peers. Iran J Child Neurol 2014;8(2):29-33.

[20] Shiari R, Saeidifard F, Zahed G. Evaluation of the prevalence of joint laxity in children with attention deficit/hyperactivity disorder. Ann Paediatr Rheum 2013;2(2):78-80.

[21] Roman T, Rohde LA, Hutz MH. A role of neurotransmission and neurodevelopment in Attention deficit hyperactivity disorder. Genome Med 2009;1(11):107.

[22] Schaaf CP, Goin-Kochel RP, Nowell KP, et al. Expanding the clinical spectrum of the $16 \mathrm{p} 11.2$ chromosomal rearrangements: three patients with syringomyelia. Eur J Hum Genet 2011;19(2):152-6.

[23] Qiu MG, Ye Z, Li QY, et al. Changes of brain structure and function in ADHD children. Brain Topogr 2011;24(34):243-52. 